\title{
Lecturas polifónicas sobre las violencias urbanas: estado del arte sobre investigaciones hechas en Medellín entre el 2010 y el $2015^{1}$
}

\author{
Polyphonic Readings of Urban Violence: \\ State of the Art on Research's Done in \\ Medellin Between 2010 and 2015
}

\section{Leituras polifônicas das violências urbanas: estado da arte sobre pesquisas feitas em Medellín, entre 2010 e 2015}

\author{
Irene Piedrahita Arcila² \\ Instituto de Estudios Políticos Universidad de Antioquia \\ Grupo de investigación Hegemonía, Guerras y Conflicto \\ irene.piedrahita@udea.edu.co \\ Max Yuri Gil Ramírez \\ Corporación Región \\ Grupo de investigación Ciudadanías, Conflicto y Políticas Sociales \\ maxgilram@gmail.com
}

Recibido: 15/05/2016

Aprobado: $28 / 10 / 2016$

1 Este texto se deriva de un estado del arte construido en el marco del proyecto Diseño de la metodología de abordaje para la formulación de una estrategia de garantías de no repetición en la ciudad de Medellín y sobre su ruta de implementación, ejecutado el primer semestre de 2015 por la Corporación Región, Financiado por USAID-OIM, Alcaldía de Medellín. Contrato de prestación de servicios pspj-2134 de 2015. Visp-0099.

2 Antropóloga y Magister en Ciencia Política.

3 Sociólogo y Magister en Ciencia Política. Estudiante de Doctorado en Ciencias Humanas y Sociales. 


\title{
Resumen
}

En este artículo se caracteriza la producción académica realizada entre el 2010 y el 2015 sobre las dinámicas de violencia urbana en Medellín. Para ello se identifican los principales enfoques teóricos de las investigaciones, las metodologías utilizadas y las hipótesis explicativas más recurrentes. así como las variables en términos de actores, modalidades de victimización, territorios y poblaciones que más han sufrido el impacto de las violencias y formas de resistencia civil no armada. Al final se establecen algunas líneas de acción para trabajos futuros en temas como afectaciones a grupos poblacionales y sectores sociales, papel de los medios de comunicación y construcción de representaciones, relación entre conflicto político armado y dinámicas criminales, y resistencias ciudadanas. Finalmente, se resalta la necesidad de más trabajos comparativos.

Palabras clave: violencia urbana; investigaciones sobre violencia; conflicto urbano; Medellín.

\begin{abstract}
This article presents a characterization of academic production done about the dynamics of urban violence in Medellin between 2010 and 2015. For this purpose, the main theoretical approaches present in research, the methodologies used and the most recurrent explanatory hypotheses are identified. The variables in terms of actors, modalities of victimization, territories and populations that have suffered the impact of violence and forms of unarmed civil resistance are also identified. At the end, some topics for future work are established on issues such as: damages to population groups and social sectors; the role of the media and construction of representations; the relationship between armed political conflict and criminal dynamics; and citizens' resistance. The study also identifies the need for establishing more comparative work.
\end{abstract}

Keywords: Urban violence; Researches on violence; Urban conflict; Medellín.

\section{Resumo}

Este artigo apresenta uma caracterização da produção acadêmica entre 2010 e 2015 sobre a dinâmica da violência urbana em Medellín. Assim, são identificadas as principais abordagens teóricas presentes nas pesquisas, as metodologias utilizadas e as hipóteses explicativas mais recorrentes. Igualmente, se identificaram as variáveis em termos de atores, modalidades de vitimização, territórios e populações que sofreram o impacto da violência e formas de resistência civil desarmada. No final se estabelecem algumas linhas de trabalho futuro em questões como danos a grupos populacionais e setores sociais, o papel dos meios de comunicação e construção de representações, a relação entre o conflito político armado e dinâmica criminal, a resistência dos cidadãos, e a necessidade de estabelecer mais trabalho comparativo.

Palavras chave: Violência urbana; Pesquisas sobre violência; Conflito urbano; Medellín. 


\section{Presentación}

Las ciudades colombianas han adquirido un protagonismo creciente en los últimos 50 años en la historia nacional, el cual es proporcional a la transformación demográfica que ha sufrido el país, que ya en 2012 mostraba que cerca de $74 \%$ de la población habitaba en las ciudades (Universidad del Rosario, 2012). En ellas se expresan con crudeza algunos de los peores males de nuestra sociedad, relacionados con problemas de pobreza extrema, exclusión, discriminación y múltiples modalidades de violencia, desde aquella que protagonizan los grupos armados vinculados al conflicto armado nacional, la que ejercen grupos de delincuencia común y redes de narcotráfico, así como problemas originados en las violencias intrafamiliar, sexual, de género, y las relacionadas con la convivencia ciudadana 4 .

La proliferación de conflictos violentos ha hecho que desde la década de 1980 las ciudades sean un epicentro para la producción de investigaciones que explican lo que acontece en sus territorios. No obstante, aun cuando existen varios documentos sobre la situación de las ciudades y en especial sobre las dinámicas de violencia que allí se han desarrollado, estamos lejos del lugar común que afirma que las ciudades están sobrediagnosticadas 5 . El caso de Medellín que abordaremos en este artículo es una muestra de ello.

Desde la década de 1980 se ha acumulado un gran número de investigaciones sobre Medellín, debido a la violencia ejercida tanto por grupos de delincuencia común y del narcotráfico, como por actores del conflicto armado, fuerza pública, paramilitares, milicias guerrilleras y, en no pocas ocasiones, mezclas inestables y explosivas entre varios de ellos. Pero, a pesar de la proliferación de producciones desde varios enfoques conceptuales y múltiples estrategias metodológicas, aún hay vetas de análisis que se pueden explorar para explicar lo que ha sucedido y sucede en la ciudad, máxime si las pensamos en el marco de los procesos de paz con los grupos guerrilleros del país, y en el posible escenario de posacuerdo que se concretaría en caso de firmarse acuerdos de paz con las guerrillas de las FARC y el ELN ${ }^{6}$.

El presente artículo tiene como objetivo realizar un balance de las publicaciones académicas que se han hecho sobre Medellín entre el 2010 y el 2015, relacionadas con violencias urbanas?. El texto se deriva de un estado del arte construido en el marco de la identificación de elementos de un Plan de Garantías de No Repetición para la ciudad de Medellín, producto de un contrato de consultoría

4 Un ejemplo de la importancia del análisis sobre las violencias urbanas se encuentra en Perea (2016), y en el Centro Nacional de Memoria Histórica (2015). Estos trabajos piensan lo que sucede con las violencias en las ciudades; el informe de memoria histórica del Centro Nacional de Memoria Histórica (CNMH) se concentra particularmente en la limpieza social.

5 Una reflexión similar se encuentra en el libro de Carlos Mario Perea (2016).

6 En el momento de escritura de este artículo ambos procesos de paz están en desarrollo. En el caso del proceso con las FARC, el acuerdo firmado en Cartagena el 26 de septiembre de 2016 está siendo renegociado por los resultados del plebiscito el pasado 2 de octubre de 2016. En el caso del ELN las negociaciones están por iniciar su fase pública a desarrollarse en Ecuador.

7 En el trabajo se retoman algunas referencias de los años 2008 y 2009 por su validez y relación con algunas de las hipótesis de trabajo sostenidas por investigaciones realizadas a partir del 2010. Este es el caso de los textos de Blair et al. (2008), el de Blair, Grisales y Muñoz (2009), y el de Max Gil (2009). Así mismo, se incluyen trabajos publicados antes del 2010, pero que refuerzan algunas conclusiones, como el texto de María Teresa Uribe de Hincapié (2002), "La investigación social en tiempos de guerra". 
establecido entre la Organización Internacional para las Migraciones (OIM) y la Alcaldía de Medellín, con la Corporación Región, una ONG con amplia trayectoria en las investigaciones sobre las dinámicas de violencia en la ciudad.

El periodo de análisis seleccionado entre 2010 y 2015 tiene dos razones. Por un lado, partimos de un estado del arte realizado por Ana María Jaramillo, investigadora de la Corporación Región ${ }^{8}$, quien retomó publicaciones hechas en Medellín entre 1980 y 2009. Por otro lado, consideramos que las investigaciones producidas entre 2010 y 2015 retoman otros puntos de análisis que son importantes para comprender lo que ha pasado en Medellín, como las especificidades en modalidades de victimización, el énfasis en formas de resistencia, las lecturas desde lo micro, lo cotidiano y lo vivencial, y las nuevas miradas a procesos vividos en la década de 1990, como en el caso de los procesos de desarme, desmovilización y reintegración (DDR) de las milicias populares.

Hoy, cuando Colombia se encuentra ad portas de un proceso de apertura democrática y modernización institucional propiciado por las negociaciones entre el Gobierno nacional y las guerrillas de las FARC y el ELN, es fundamental el rol de las ciudades, tanto de cara a la implementación de los acuerdos de paz como a asumir transformaciones aplazadas por las urgencias de la confrontación armada. Si bien es cierto que el tema urbano no está de manera explícita en las agendas de negociación, y que los principales fenómenos de violencia urbana hoy no están relacionados de manera directa con el conflicto armado, es fundamental repensar las ciudades para la fase más inmediata del posacuerdo, y para prevenir nuevas dinámicas de violencia que pueden obstaculizar el proyecto democratizador que se pretende abrir en el futuro inmediato. La paz y la democracia en las ciudades serán componentes imprescindibles en la tarea de construir un mejor país y para ello se requiere el aporte de los centros de investigación académica y social. Esa es una de las finalidades de este artículo.

Para la elaboración de este texto se identificaron libros, capítulos de libro, ponencias y artículos en revistas especializadas de las principales universidades del país donde se tenía referencia de publicaciones hechas sobre la ciudad. Así mismo, se revisaron las bases de datos Redalyc, Dialnet y JStore, algunos materiales producidos desde las distintas secretarías de la Alcaldía de Medellín, y trabajos de grado que abordan las violencias urbanas. Se identificaron 117 materiales que fueron incluidos en una base de datos para su organización y posterior priorización. Para el análisis se eligieron cincuenta textos para ser revisados de forma detallada, identificando enfoques teóricos y metodológicos, así como algunas de las interpretaciones más importantes.

Este artículo está dividido en cuatro partes: en la primera se abordan los enfoques teóricos más usados por los investigadores. En la segunda, las metodologías y leguajes utilizados. En la tercera se referencian las principales temáticas abordadas para dar cuenta de las violencias que ha vivido Medellín y, al final, en el apartado de cierre se sugieren algunas líneas de investigación a desarrollar en el futuro. Esperamos que con este texto se abra una discusión para seguir

8 Una versión digital de dicho documento se encuentra en la web de la Corporación Región. http:// www.region.org.co/images/publicamos/documentos/Acerca_de_los_estudios_sobre_conflicto_ armado_y_violencia_urbana_en_Medellin.pdf 
repensando la ciudad y para establecer nuevos puntos de análisis y debate en la comunidad política y académica de Medellín.

\section{1. ¿Cómo teorizar las violencias de la ciudad? Enfoques conceptuales para abordar las violencias urbanas en Medellín}

La producción sobre violencia y conflicto armado en el país se ha centrado en la comprensión y explicación de su permanencia espaciotemporal. Dicha producción tiene un cambio particular en la década de 1980, cuando las ciudades empiezan a ser protagonistas en la historia política nacional por el incremento de los eventos violentos. Lo acontecido en esa década exige que los investigadores se preocupen por comprender los fenómenos urbanos, donde modalidades de victimización como asesinatos selectivos y atentados con carro-bombas, "motivaron la producción de un gran número de trabajos principalmente de corte sociológico y sicológico que analizaban el ejercicio de la violencia en los contextos urbanos, especialmente en la juventud" (Zapata, 2012, p. 25).

Las particularidades de los conflictos violentos y sus actores, la entrada en escena de nuevos territorios y el incremento de estadísticas sobre homicidios incentivaron la producción académica sobre violencias urbanas, llevando a la construcción de enfoques teórico-metodológicos para comprender lo que sucedía. Tal como proponen algunos investigadores del Instituto de Estudios Políticos (IEP) de la Universidad de Antioquia, para finales de la década de 1980 las violencias producidas en el marco de lo urbano ya eran un tema de constante atención por parte de la academia colombiana (Restrepo, Pérez y Vélez, 2012a).

La producción académica sobre violencias urbanas se vio alentada, entre otras cosas, porque el informe Colombia: Violencia y democracia, publicado en 1987, ya alertaba que "el mayor número de muertos en el país se estaba produciendo en las áreas urbanas, indicando un cambio en su dinámica, que hasta el decenio de 1970 era fundamentalmente rural" (Restrepo et al., 2012a, p. 16). En dicho informe, además, se resaltaba que el caso de Medellín era particular no solo por el número de muertos, sino por "la intensidad, la generalización y la complejidad del fenómeno" (Restrepo et al., 2012a, p. 18).

Ana María Jaramillo (s.f.) retoma en su estado del arte dos enfoques teóricos enmarcados en la relación conflicto-violencia: el conflicto urbano y la violencia urbana. El concepto de violencia urbana sigue teniendo vigencia en la mayoría de los estudios realizados sobre Medellín entre 2010 y 2015, aun cuando se han hecho algunas salvedades respecto a su uso'. Pero, en el caso del uso del concepto conflicto urbano, este se ha cuestionado desde diferentes puntos y su uso ha menguado considerablemente, puesto que se considera que, desde algunas perspectivas de lo urbano, la ciudad es vista como un entorno "vacío" en el que

9 Nos referimos a que algunas investigaciones prefieren hablar de violencias asociadas al conflicto armado para especificar la conexión con el ámbito nacional. Algunos investigadores también han cuestionado el uso de violencia urbana porque se termina incluyendo en un mismo conjunto violencias de diverso tipo. No obstante, a falta de otros conceptos, "violencia urbana" sigue siendo un término ampliamente usado por los sectores académicos y sociales de la ciudad. 
la violencia llega desde afuera. También se considera que se estigmatizan formas de conflicto que son inherentes a la vida en sociedad. De hecho, el carácter ampliado de conflicto y la noción vacía de ciudad fueron, desde principios de la década del 2000, dos cuestionamientos fuertes que se recogieron en las investigaciones producidas posteriormente.

Algunos investigadores postulan que no debe asimilarse la violencia urbana con el conflicto urbano, pues esto "supone una concepción negativa del conflicto como expresión de la diferencia y, así mismo, conlleva a una estigmatización bajo el campo de acción violenta de todo tipo de luchas sociales por la construcción y reorganización de la ciudad" (Gil, 2009, pp. 66-67). Por otro lado, el concepto de conflicto urbano, como ya lo anotaba Jaramillo retomando a Vilma Liliana Franco, establece una visión de la ciudad espacialista en la que

\footnotetext{
[...] se asocia la ciudad con el espacio físico medible, limitable, visible y demográficamente denso, definido en oposición a lo rural, dejando de lado una aproximación a lo urbano no como simple conglomerado de casas, edificios, calles, espacios públicos, etc., sino también como el eje de procesos económicos, sociales, políticos y culturales diferentes a los de la vida campesina, y en los cuales se anudan distintas formas de conflictividad que no necesariamente son resueltas mediante la apelación de la violencia (Franco citado por Jaramillo, s.f., p. 8).
}

Esta forma espacialista de comprender la ciudad otorga una caracterización de la misma como una suerte de contenedor para llenar, donde se omiten los acumulados previos de los barrios, las dinámicas propias de conflictividad y las historias de poblamiento y desarrollo de las ciudades. Así mismo, esconde formas de territorialidad particulares, tendiendo a asimilar el conflicto con condiciones estructurales de pobreza y desigualdad, cayendo en estigmatizaciones nocivas para territorios considerados o clasificados como marginales, empobrecidos o excluidos (Franco, 2000; Restrepo et al., 2012a).

Lo anterior fue rápidamente cuestionado por los investigadores de la ciudad, puesto que resultaba evidente que las dinámicas de violencia no eran una particularidad de los estratos bajos, y que las periferias no eran las únicas con estadísticas violentas. Se planteaba entonces que "no se puede decir que la violencia [...] sea específica y excluyente de un espacio concreto de la ciudad, sino, por el contrario, que se va moviendo indistintamente, incluyendo de manera peculiar, el denominado 'centro de la ciudad' [...]" (Restrepo, Pérez, Vélez, 2012b, p. 105).

Uno de los trabajos que aporta lecturas socio-espaciales sobre la ciudad y sus violencias es la investigación De memorias y de guerras (Blair et al., 2008) realizada en el Instituto de Estudios Regionales (INER) de la Universidad de Antioquia ${ }^{10}$. En dicho trabajo las investigadoras proponen un análisis de corte socio-espacial para entender las violencias urbanas, donde la geopolítica crítica, con aportes de autores como John Agnew y Ulrich Oslender, resultan enriquecedores para

10 Si bien esta publicación es previa al periodo de tiempo elegido para este artículo, su divulgación como producto de investigación tomó fuerza en la ciudad a partir del 2010, cuando nuevos enfoques aparecieron en la mira de los centros de investigación de Medellín. Eso se pudo constatar a partir de entrevistas y conversaciones complementarias que se realizaron para la construcción del estado del arte. 
esta mirada. Lo más significativo son los cuestionamientos hechos al concepto de conflicto urbano y de guerra urbana, pues consideran que son insuficientes para explicar las dinámicas propias de la violencia en Medellín. Para las autoras estos conceptos reducen lo que sucede en la ciudad a una expresión del conflicto armado nacional, dejando por fuera manifestaciones territoriales endógenas que producen otros fenómenos violentos. En sus palabras

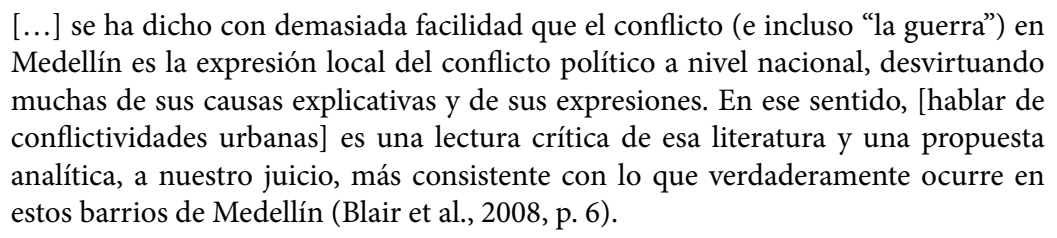

La categoría de conflictividades urbanas se constituye en una crítica al desconocimiento que ciertas lecturas hacen de las dinámicas barriales y la existencia de otros conflictos que no necesariamente están asociados con los actores armados protagonistas de lo nacional. Si bien no desconocen que a los barrios llegan los "actores de la guerra", establecen que su entrada se conecta con "dinámicas barriales preexistentes" que particularizan las maneras en que se desarrolla la confrontación. Esta categoría resulta ser una nueva veta analítica para comprender lo acontecido en la ciudad, pues evidencia que Medellín ha tenido historias particulares que deben reconocerse para comprender en extenso la magnitud y dinámicas de las violencias urbanas"

Otro concepto utilizado en investigaciones recientes es el de orden violento, trabajado especialmente por Carlos Mario Perea (2014). Los órdenes han sido estudiados por diversos autores para entender cómo en un territorio confluye una serie de ordenamientos jurídicos, sociales y culturales que configuran la vida social; esto a través de preguntas tipo “¿cómo emerge el orden, cómo es sostenido, cuestionado, destruido, transformado y recreado?" (Kalyvas citado por García, 2011, p. 60). La relación órdenes-violencia se define "como un proceso social en el cual las acciones violentas moldean nuevas estructuras y nuevas formas de comportamiento, y por esa vía, redefinen las formas de la organización societal" (García, 2011, p. 62).

En un trabajo comparativo entre Medellín y Río de Janeiro, Perea (2014) usa el concepto de orden violento para mostrar cómo "el orden de la vida en estos contextos [urbanos] supone que la inserción de los agentes violentos se produce mediante un complejo intercambio con las formas sociales propias de las barriadas periféricas, un intercambio regido por la violencia" (p. 4) y, por tanto, las maneras en que se regula la vida social se dan por "el encuadramiento simbólico de la muerte cercana". Este, según Perea (2014):

[...] es un orden violento, fundado en el pánico universal a una muerte que puede estar a la vuelta de la esquina o en la puerta de la casa. Es cercana no sólo porque los

ll Una hipótesis de trabajo podría ser que esto es particular de todas las ciudades en el país, y aunque ciudades como Cali, Medellín, Bogotá y Barrancabermeja contaron con formas de violencia similares por la participación de actores armados del conflicto nacional, las expresiones y efectos no fueron las mismas. En las conclusiones del texto, proponemos, precisamente, la necesidad de realizar estudios comparados donde se ponga a prueba este tipo de conceptos. 


\begin{abstract}
agentes están ahí, en la calle del lado, sino porque su administración es arbitraria: las personas sobre quienes puede recaer no participan ni de las reglas de constitución y menos de las formas de aplicación de la violencia. La obediencia está garantizada (p. 7).
\end{abstract}

En relación con los conceptos mencionados, existe una serie de enfoques teóricos con los que se han comprendido las violencias en la ciudad. Zapata (2012) explica que la violencia en Colombia generalmente ha sido entendida bajo dos enfoques analíticos. Por un lado, hay una fuerte asociación de la violencia como fenómeno político, "en donde ésta es la expresión de los conflictos ideológicos que encarnan las confrontaciones por los proyectos de Estado nación y los modelos de desarrollo" (Zapata, 2012, p. 11). Este enfoque, por lo general, establece un análisis macro de los conflictos y violencias vividos en el país desde el siglo XIX para desembocar en el conflicto social y armado que vive el país actualmente. Por otro lado, existe un campo analítico que comprende la violencia como "un fenómeno social [que] se expresa en las relaciones cotidianas que no están mediadas necesariamente por motivaciones ideológicas, pero que sí parten de las necesidades y las dinámicas sociales y económicas que se dan en determinados territorios" (Zapata, 2012, p. 11). En este campo se incluyen violencias que no están ligadas de modo específico con las confrontaciones con el Estado' ${ }^{12}$, específicamente "la violencia juvenil, la violencia urbana, la violencia intrafamiliar, la criminalidad organizada, el negocio de la protección ilegal, la delincuencia común, el narcotráfico, entre otros tipos de violencia [...]" (Zapata, 2012, p. 11).

Desde la década del 2000 también hay cuestionamientos a algunas lecturas sobre la violencia en la ciudad en las que se separa la política de aquellas formas de la violencia que estaban alejadas o eran difíciles de emparentar con lo sucedido en el ámbito nacional (Nieto y Robledo, 2006; Riaño, 2006; Alonso, Giraldo y Sierra, 2006; Blair et al., 2008). Estos aportes logran que después del 2010 haya un resurgir analítico en las interpretaciones sobre las violencias urbanas, donde las cotidianidades de la violencia, las acciones de resistencia y las lecturas desde la memoria cobran un papel protagónico, conjugando los dos enfoques que identifica Zapata.

El libro Violencia y subjetividad. Narrativas desde la vida cotidiana de Berrío, Grisales y Osorio (2011) es una apuesta teórica por entender las conflictividades urbanas desde la cotidianidad como un "enfoque espacio-temporal interpretativo" que posibilita "comprender y analizar la violencia en relación con las subjetividades y las vivencias propias de los individuos que han padecido -y padecensituaciones de violencia en la ciudad" (Berrío, et al., 2011, p. 86). Este enfoque es alimentado con un contexto histórico de las conflictividades urbanas, que definen como "otra clave' para leer el conflicto y la continuidad de la violencia en la vida diaria" (Berrío et al., 2011, p. 86).

Por su parte, Catalina Tabares invita a pensar a las víctimas de violencias urbanas desde las subjetividades, pues es un "eje articulador que permite construir

12 Aunque desde la lectura que hacemos para este artículo, reconocemos que no solo son confrontaciones contra el Estado, sino también violencias institucionales para mantener un orden. Al respecto, ver los trabajos de Camilo Yarce (2013) y Daniel Villegas (2014) revisados para la construcción del estado del arte del que se deriva este artículo. 
la realidad social y comprender [su] devenir sujeto político" (Tabares, 201l, p. 17). En estos argumentos la cotidianidad juega un papel fundamental. Este es uno de los aportes más significativos de este tipo de investigaciones, donde lo micro, lo subjetivo y lo vivencial se dimensionan como elementos explicativos para comprender lo que ha sucedido en Medellín en los últimos años.

En general, los investigadores han explicado la violencia en Medellín partiendo del hecho de que es un fenómeno mucho más complejo que la confrontación entre actores y que, por ello, habría que revisar aprendizajes, mutaciones, dinámicas de relacionamiento y disputas entre ellos. Lo que se propone es que la violencia en Medellín responde a dinámicas particulares de poblamiento y configuración territorial que, articuladas a procesos cotidianos, singulariza lo que acontece en la ciudad y, por supuesto, aquellas lecturas que pueden hacerse sobre sus violencias.

\section{2. ¿Cómo aprehender Medellín? Enfoques metodológicos para abordar las violencias urbanas en la ciudad}

La mayoría de los enfoques metodológicos para comprender lo que ha sucedido en Medellín se centran en lo cualitativo, combinado en algunos momentos con estadísticas para conocer "la magnitud de los fenómenos estudiados, sobre todo, en los trabajos relacionados con la [violencia] sexual y el homicidio" (Jaramillo, s.f., p. 10). El enfoque cualitativo ha sido la referencia principal para conocer de primera mano los hechos violentos acontecidos en la ciudad, pues herramientas como las entrevistas en profundidad, los recorridos por barrios, comunas y lugares de memoria, la cartografía social, los talleres de memoria y grupos focales, buscan entender, desde lo cotidiano y lo subjetivo, las tramas de la violencia más allá del dato estadístico.

Los estudios de corte subjetivo, predominantes en los últimos años, optan por el testimonio como una herramienta metodológica y política para resaltar el papel activo de las víctimas (Tabares, 2011). Quienes utilizan los testimonios como estrategia metodológica aseveran que con estos los "sujetos-sufrientes despliegan las dimensiones subjetivas, espaciales y temporales vividas en la violencia, y [...], de una u otra manera, permiten relativizar las periodizaciones y los discursos desde los cuales se ha explicado la violencia en la ciudad" (Berrío et al., 2011, p. 86). Esto, ligado a la historización del relato, articula los testimonios a unas violencias más amplias, superando, de algún modo, la dicotomía entre lo micro y lo macro presente en algunas investigaciones.

Así mismo, el boom de la memoria del que habla la investigadora Sandra Arenas (2012) para los últimos quince años en el país también ha permeado los trabajos académicos de la ciudad y ha posibilitado la inclusión de otro tipo de lenguajes al ejercicio investigativo. Desde el periodismo, por ejemplo, ha habido un aporte significativo al proponer otras maneras de narrar las violencias urbanas ${ }^{13}$. Patricia Nieto (2013) se pregunta "cómo narrar la guerra desde el adentro

13 Aun cuando no son textos derivados de investigaciones de las ciencias sociales, en el periódico De La Urbe de la Facultad de Comunicaciones de la Universidad de Antioquia, son evidentes nuevos lenguajes para narrar lo acontecido en la ciudad. 
sin aceptar que una investigación en tal sentido logra su demarcación más en torno al objeto mismo que a un método predeterminado por una disciplina específica" (p. 233). Esto se logra a partir de la transdisciplinariedad, en la medida en que "el objeto de observación (las narrativas) que es a la vez objetivo (narrar), al convertirse en una obra por construir, se ubica en esa generosa intersección donde diversas disciplinas se rozan, se cruzan y se fertilizan: la producción cultural" (Nieto, 2013, p. 233). La memoria como herramienta y enfoque metodológico, que cada vez y con más fuerza se une como parte vital de las investigaciones, no es propiedad de un campo específico del saber, sino que es apropiada por distintas disciplinas e investigaciones ${ }^{14}$. Así mismo, la generalidad en los trabajos sobre violencias en Medellín se centra en enfoques interdisciplinares.

Adicional a la proliferación de enfoques cualitativos, en las investigaciones entre 2010 y 2015 también hay un creciente interés por llenar de contenido los análisis estadísticos para comprender las violencias urbanas. Esto es importante porque suele criticarse lo peligrosas que resultan las estadísticas para estigmatizar o señalar ciertos grupos y sectores de la ciudad. Sin embargo, las dificultades humanas y analíticas de las estadísticas no las demeritan, sino que, por el contrario, exigen mayor pericia del investigador. Un ejemplo de uso alternativo de las estadísticas lo da la Secretaría de la Juventud (2015) en el libro Carrera de obstáculos, crecer y resistir. Relatos sobre seguridad y convivencia juvenil en Medellín. En este texto se mezcla un ejercicio riguroso de investigación cuantitativa-cualitativa con un lenguaje llamativo, donde las estadísticas son complementadas y leídas bajo el prisma de lo cualitativo.

Uno de los vacíos o campos metodológicos por explorar en los estudios sobre Medellín consiste en el poco uso del método comparado. Dentro de lo identificado entre 2010 y 2015 está el trabajo de Ana María Jaramillo y Carlos Mario Perea (2014) quienes, desde la Corporación Región y el Instituto de Estudios Políticos y Relaciones Internacionales (IEPRI) de la Universidad Nacional de Colombia, con el auspicio del International Development Research Centre (IDRC), realizaron un trabajo con una mirada comparada entre los fenómenos violentos de Medellín, Bogotá, Río de Janeiro y Ciudad Juárez. De acuerdo con los autores,

\footnotetext{
La mirada comparativa $[\ldots]$ se mueve entonces entre aquello que arma la diferencia y aquello que funciona como denominador común. En otras palabras, la mirada comparativa ha de dar cuenta de las marcadas diferencias entre las ciudades y sus actores, pero bajo la premisa de determinar la singular gestión del narcotráfico adoptada en cada ciudad (Jaramillo y Perea, 2014, p. 11).
}

En términos metodológicos es importante decir que en los últimos años ha habido un creciente interés por reconocer otro tipo de lenguajes y de formas de producción de conocimiento sobre las violencias urbanas. Este es el caso de los grafitis, la música -particularmente el rap-, la fotografía y los ejercicios

14 Otro trabajo que ilustra lo anterior es el artículo escrito por Pérez, Aristizábal, Ríos y Osorno (2014). En el texto se hace una revisión del poblamiento y las dinámicas de los barrios La Honda, La Cruz y Bello Oriente, a partir de los enfoques de la memoria y la historia reciente. En este trabajo se involucran, además, interpretaciones sobre violencias y resistencias vividas en estos barrios. 
audiovisuales, en los que se plasman de otras maneras las violencias en la ciudad. Colectivos como Ciudad Comuna, el Retorno Comunicaciones y la Corporación Pasolini en Medellín, entre otros, han apostado por formas alternativas de narrar las conflictividades urbanas y las violencias a partir de documentales y cortometrajes ${ }^{15}$. Este tipo de lenguajes se expresan como piezas individuales -documentales, cortometrajes- pero también en productos alternativos para divulgar los resultados de las investigaciones.

Finalmente, dentro de las formas como aprehendemos lo que sucede en la ciudad, está el papel que ocupan los investigadores frente a las violencias urbanas. Si bien Ana María Jaramillo resalta en su estado del arte que en algunos estudios entre 1980 y el 2009 se indagó "por la responsabilidad social del investigador, las implicaciones de las intervenciones investigativas sobre los sujetos mismos, y la manera como repercuten en el mismo investigador" (s.f., p. 10), consideramos que este es un campo que puede explorarse aún más, pues las reflexiones sobre lo que implica estudiar las violencias urbanas son pocas. Al respecto, valdría la pena pensar qué significa lo que ya María Teresa Uribe de Hincapié llamó "la investigación social en tiempos de guerra" (2002) y lo que conlleva para los investigadores. Dichos ejercicios, además de que aportan al carácter reflexivo de los escenarios de producción de conocimiento, sirven de referencia para otros investigadores que decidan abordar el tema, y para generar relaciones horizontales y menos nocivas con las comunidades y sujetos con los que se trabaja.

\section{3. ¿De qué hablamos cuando hablamos de violencias urbanas? Periodizaciones, modalidades y respuestas}

Buena parte de las investigaciones que se han producido en Medellín proponen la década de 1980 como "punto de inflexión en el escalamiento del conflicto armado" (Jaramillo, s.f., p. 11), pues en ese momento hay una "expansión de la guerrilla y [una] consolidación de una economía ilegal de la droga, que se considera determinante en un aumento de la violencia sin precedentes [...]" (Jaramillo, s.f., p. ll). En efecto, proponer hitos coyunturales en la historia de las violencias urbanas ha sido una de las preocupaciones de muchas investigaciones, lo que se evidencia en el interés por periodizar dichas violencias y establecer una cronología para contextualizar los actores y comprender matices históricos, dinámicas del conflicto y modalidades de victimización.

La mayoría de las periodizaciones que se proponen para Medellín señalan que la década de 1980 se caracteriza por el auge de violencias asociadas al narcotráfico. Entre 1991 y 1994 se referencia la consolidación de las milicias locales en la ciudad, seguidas por los respectivos procesos de desmovilización que firmaron con el gobierno local en abril de 1994 (Giraldo y Mesa, 2013). Entre 1995 y el 2005 se identifica que hay un escalamiento del conflicto armado nacional, el cual llega a las ciudades a través de la presencia de grupos paramilitares

15 Piezas como El juego de la vida realizado por Pasolini en Medellín o La dignidad que emerge de las laderas hecho por Ciudad Comuna, son ejemplos de otras formas de producir conocimiento alrededor de la ciudad, sus historias y sus violencias. 
asociados a las Autodefensas Unidas de Colombia (AUC) ${ }^{16}$, y de milicias urbanas de las FARC y el ELN. Finalmente, entre el 2005 y el 2010 hay un reordenamiento de las violencias, a partir de la desmovilización de los paramilitares entre 2003 y 2005, y la presencia de nuevos actores y formas de accionar (Jaramillo, s.f.).

Otro ejemplo de cronologías para entender lo que ha sucedido en la ciudad lo dan Blair et al., (2008) y Blair et al. (2009), quienes definen tres periodos históricos de las conflictividades urbanas, partiendo del ingreso y la recomposición de los actores de la violencia. Hay un momento de antecedentes entre 1980 y 1994, caracterizado en sus primeros años por el "fenómeno del narcotráfico y [el] sicariato, [mientras que] los últimos años del periodo están caracterizados por la presencia de las bandas y los inicios del fenómeno miliciano" (Blair et al., 2008, p. 143). El segundo momento, conflicto reciente, se da entre 1995 y el 2005, caracterizado "por la presencia de "actores políticos" en la ciudad y la confrontación más aguda entre los diversos actores de la guerra" (Blair et al., 2008, p. 143). El tercer momento es la situación actual, entre 2005 y 2008, fecha en la que se realizó el estudio, donde aparecen "dinámicas más coyunturales como las negociaciones con los grupos paramilitares pero, sobre todo, las "audiencias" con los jefes [...]" (Blair et al., 2008, p. 144). En general, esta periodización tiene un interés particular por evidenciar cómo las problemáticas previas de los barrios -esto es, las conflictividades urbanas- se articulan en distintos momentos con la llegada o la resignificación de actores que hacen parte del conflicto armado nacional, como las milicias urbanas de los grupos guerrilleros y las AUC.

Gerard Martin (2014), por su parte, establece una cronología más amplia, proponiendo un contexto general de la ciudad y su conformación histórica, para mostrar cómo hay antecedentes de la economía criminal en Medellín desde la década de 1950. Para el autor, "Medellín, durante los años cincuenta y sesenta, se consolidó, entre muchos otros desarrollos, como un importante centro de contrabando, tanto de exportación (café) como de importación (cigarrillos, licor, ropa y productos de lujo)" (Martin, 2014, p. 63). La lectura de Martin está cruzada por un detallado recuento de lo sucedido en la ciudad en términos criminales y violentos, pero también por la descripción de muchas de las iniciativas sociales, comunitarias e institucionales para detener la violencia o mitigar sus impactos, lo que se constituye en un insumo fundamental para analizar las formas de respuesta de la ciudad a los fenómenos violentos.

El libro de Martin pone en evidencia la necesidad de estudiar las maneras en que la institucionalidad local ha respondido a las violencias urbanas, no como una apología a la administración municipal, sino para comprender las formas en que el Estado se resignifica para atender las coyunturas particulares con las que se enfrenta. El escenario de Medellín se perfila como un lugar idóneo para analizar las transformaciones institucionales, la inclusión de agendas de trabajo para mitigar y resolver los problemas violentos de la ciudad, y la generación de políticas públicas relacionadas con formas de respuesta a las violencias urbanas.

De la lectura cronológica de las violencias urbanas se recogen conclusiones que van más allá de los periodos o momentos, y que apuntan a factores de Granada. 
explicativos de la violencia (Jaramillo, s.f.). Estos se piensan en función de una multicausalidad de la violencia urbana articulada a los procesos de modernización e industrialización de la ciudad de Medellín, y las cronologías más recientes, como las presentadas en este artículo, aluden a comprender lo sucedido en la ciudad desde una perspectiva escalonada con lo nacional, sin que ninguna de las dos escalas -local/nacional- sea hegemónica.

Las lecturas de las violencias urbanas, además, se asocian a visiones sociopolíticas, donde se ubica como "principal causa de la violencia, a la precariedad del Estado en el cumplimiento de sus funciones como garante del orden social" (Vergara, 2013, p. 3). Esto, no obstante, también se articula con procesos sociales propios de los fenómenos urbanos donde resultan fundamentales "las acciones ilegales del narcotráfico y cuyos requerimientos para su durabilidad, van desde la creación de fuertes estructuras criminales, hasta la formación de redes de intermediación con actores sociales y políticos del campo institucional legal urbano" (Vergara, 2013, p. 3).

Después de 2010 son pocas las investigaciones que se centran en analizar las causas de las violencias urbanas, salvo trabajos como el de Martin (2014) y Perea (2014), o la lectura comparada que hacen Jaramillo y Perea (2014). En estos tres trabajos son comunes las hipótesis que construyen en torno al narcotráfico y su impacto sobre las dinámicas de violencia urbana, es decir, el potencial del narcotráfico como análisis explicativo de lo ocurrido en Medellín.

El trabajo de Martin (2014) establece como eje de la violencia, la existencia de poderosas organizaciones dedicadas al narcotráfico, las cuales sirven como potenciadoras de otros fenómenos, como la débil presencia del Estado en el territorio, los graves procesos de corrupción institucional, y la proliferación de grupos armados como las bandas y los combos delincuenciales. En este contexto, también se identifica como un agente detonante de la violencia de la ciudad a los grupos guerrilleros y sus esfuerzos por hacer presencia a través de organizaciones milicianas. Martin también extiende su análisis al impacto del narcotráfico en otras áreas como la cultura:
La proliferación de bandas y la penetración de la economía del narcotráfico empezó a generar una inversión de valores y de aspiraciones en modelos de socialización juvenil barrial. [...] De manera inevitable, en los barrios donde estos fenómenos se dieron, el miedo y el silencio se instalaron, las relaciones sociales se fragmentaron y la confianza se degradó (Martin, 2014, pp. 148-149).

Por su parte, el trabajo de Carlos Mario Perea (2014) se centra en la existencia de unos grupos violentos, entendidos como actores ilegales que ejercen dominio armado sobre sectores de la ciudad, y busca responder dos preguntas: cómo se ejerce ese dominio, y cómo responde la población. Al caracterizar el origen del conflicto violento, Perea (2014) señala que "[...] tanto Río como Medellín remontan el inicio de su conflicto violento a los años ochenta del siglo pasado, cuando cada una desarrolló una gestión del negocio del narcotráfico que tras una y otra transformación se mantiene hasta hoy" (2014, p. 37).

Finalmente, en el estudio comparativo de cuatro ciudades latinoamericanas, Río de Janeiro, Medellín, Bogotá y Ciudad Juárez (Jaramillo y Perea, 2014), se parte del reconocimiento de la doble condición de las ciudades latinoamericanas, 
lo que los autores llaman la paradoja, entendida como la condición de ciudades revestidas de toda importancia, pero que, pese a su marcada preponderancia, se trata de ciudades cruzadas por criminalidades y violencias intensas (Jaramillo y Perea, 2014, p. 9). Sobre el elemento central definido para hacer la mirada comparativa este se define así

\begin{abstract}
Nuestra hipótesis, sin embargo, asume que las marcadas diferencias no dejan de permanecer conectadas por un rasgo común que las vincula. En las cuatro ciudades el negocio del narcotráfico opera como el mercado ilegal dominante, mercado del cual se derivan importantes márgenes de ganancia y se coordina otro conjunto de actividades ilegales (Jaramillo y Perea, 2014, pp. 10-11).
\end{abstract}

Aun cuando estas investigaciones significan una actualización de la evolución de la violencia en la ciudad de Medellín ligada al narcotráfico, no proponen enfoques novedosos sobre las causas, dado que este ha sido uno de los elementos que más atención ha generado en los distintos trabajos realizados sobre la ciudad. Por ello, entre 2010 y 2015 se han consolidado otros temas de interés, como las modalidades de violencia -en particular el desplazamiento forzado intraurbano-, las acciones de resistencia, los procesos de desmovilización o los análisis cotidianos sobre las violencias urbanas, y aun cuando ello no excluye alusiones a las causas de las violencias urbanas, estas, en tanto tema de investigación, no han sido centrales pues son otros los sujetos y otras las lecturas que ocupan el panorama.

En términos de modalidades de victimización, desde 1997, pero concretado en una publicación del IEP en el texto de Manuel Alonso, William Pérez y Juan Carlos Vélez (2012) sobre seguridad y violencia homicida en Medellín, así como en la investigación del Centro de Análisis Político de la Universidad EAFIT (2014), hay una relectura del homicidio como principal fuente de medición de la violencia, en la que se cuestiona que sea el elemento central a considerar en las investigaciones.

\footnotetext{
El homicidio, el conflicto, la violencia y la ciudad son más que la sumatoria mecánica de lo oculto y lo registrado sobre "a cuántos, dónde y con qué se les ha causado la muerte" o, de otra manera, el homicidio es apenas uno de los puntos de arribo -el más dramático y lamentable desde luego- de la violencia y el conflicto urbano. La reconstrucción del evento homicida parte del dato, de la cifra, pero no sólo hacia su cuantificación posterior, sino hacia la observación cualitativa de los procesos desencadenantes y desencadenados (Restrepo et al., 2012b, p. 94).
}

Redimensionar el homicidio dentro de la violencia urbana de Medellín ha posibilitado que las investigaciones estudien otro tipo de modalidades o patrones de victimización y que se revisen, además, los enfoques desde los cuales se piensa la seguridad ciudadana. De hecho, en cuanto a modalidades de violencia, una de las que se ha estudiado con fuerza desde el 2005 y que se aleja de la violencia homicida, es el desplazamiento forzado, el cual ha sido analizado en varias tesis de pregrado y posgrado, así como en investigaciones realizadas desde la ciencia política, la psicología y la sociología. Este fenómeno ha sido abordado, al menos, desde dos perspectivas. Por un lado, aquellos trabajos que analizan los efectos que ha afrontado Medellín al recibir población desplazada de distintos lugares 
del departamento y el país. Por otro, aquellos que trabajan los desplazamientos forzados intraurbanos.

El grupo de investigación en Psicología Social y Política de la Universidad de Antioquia realizó en 2014 la investigación Significación de la experiencia de pérdidas y duelos en personas desplazadas de la ciudad de Medellín ${ }^{17}$, la cual analiza los procesos de configuración de los sujetos a partir de su llegada como desplazados $^{18}$. En esta investigación se dice que el desplazamiento forzado es una "experiencia subjetiva" que "implica acoger la idea del mundo experiencial como mundo de vida" (Molina, Díaz y Marín, 2014, p. 20). Dichas experiencias se materializan con la llegada de población desplazada a la ciudad, quienes definen su encuentro con la ciudad como "un llegar a pasar necesidades" (Molina et al., 2014, p. 75), y donde "la pobreza es el contexto de la vida después del desplazamiento" (Molina et al., 2014, p. 75). De hecho, la relación difícil con la ciudad, mediada por falta de alternativas económicas, y redes y vínculos de solidaridad, trae como consecuencia nuevos desplazamientos, bien sea a otros barrios de Medellín o hacia municipios cercanos.

Los investigadores también relatan cómo aprender a vivir en la ciudad implica una serie de mecanismos de resistencia y acción que les posibilita la sobrevivencia. Al respecto, dicen que la llegada a Medellín necesita también de una serie de estrategias que permite que las personas se adapten a los nuevos contextos de un espacio desconocido, para lo que deben "asignar significado a nuevos espacios y objetos, apropiarse de ellos para encontrar la forma de "moverse": explorar las rutas, caminarse los espacios, recurrir a las indicaciones de otros, buscar lugares de referencia como edificios o monumentos" (Molina et al., 2014, p. 81).

Frente al desplazamiento forzado intraurbano, encontramos el Informe de Memoria Histórica sobre la Comuna 13 realizado en el 2011 por la Corporación Región y el Grupo de Memoria Histórica. El Informe, a partir de una serie de relatos de memoria, narra lo sucedido con el desplazamiento forzado en la Comuna 13 y enfatiza en la importancia de reconocerlo como una modalidad de victimización propia de las violencias en el marco del conflicto armado que se desplegaron en la ciudad. Uno de los logros significativos del Informe radica en el lugar en el que pone dicho desplazamiento, estableciendo un hito para las víctimas y quienes trabajan por sus derechos. Ello es importante porque aun cuando el desplazamiento forzado intraurbano no solo se ha dado en Medellín o en esta comuna, "es claro que éste constituye un fenómeno relevante en la Comuna 13

17 Esta investigación es importante porque es uno de los referentes de trabajo que tiene la psicología social en la ciudad. En una conversación sostenida con la investigadora Natalia Molina para complementar la elaboración del estado del arte, resaltó el hecho de que los trabajos de psicología relacionados con el conflicto armado aparecen en la década del 2000, abordando específicamente los impactos en el duelo. Sin embargo, son pocos los trabajos que asocian directamente la psicología con las conflictividades o violencias urbanas. Esto, según la investigadora, apenas empieza a explorarse en la disciplina y, por ello, es uno de los campos que podrán trabajarse en futuras investigaciones.

18 De esta investigación se deriva el artículo Las pérdidas y los duelos en personas afectadas por el desplazamiento forzado, publicado en el 2015. En este se sintetizan las principales conclusiones a las que llegan los investigadores y se hacen aportes significativos al entendimiento de los procesos de duelo en población desplazada. Ver Díaz, Molina y Marín (2015). 
puesto que éste es el sector de donde históricamente más se expulsa población en Medellín" (Centro Nacional de Reparación y Reconciliación, 201l, p. 49).

Algunos trabajos que han abordado el desplazamiento forzado en Medellín se han centrado en las acciones de resistencia de las comunidades para reterritorializarse en la ciudad y para resistir a los daños y las victimizaciones producidas por las violencias urbanas. Un trabajo significativo es la tesis del politólogo de la Universidad de Antioquia Jonathan Murcia (2010), quien retoma las categorías de acción colectiva y derecho a la ciudad para analizar el fenómeno del desplazamiento forzado en Medellín entre 2002 y 2009. Murcia (2010) dice que "los repertorios de acción colectiva de la población desplazada en Medellín [son] todas aquellas acciones ejecutadas por esta población orientadas hacia la reivindicación y reclamo de posibilidades reales de acceso a los derechos que les han sido vulnerados" (p. 110). Dichas acciones no se inscriben solamente en la institucionalidad, sino que son espontáneas, "dependiendo de las circunstancias y del carácter estratégico de la acción adelantada” (Murcia, 2010, p. 110).

En la misma vía se encuentra el trabajo de los investigadores James Granada y Sandra González (2009) del IEP de la Universidad de Antioquia, quienes estudian la acción colectiva en población desplazada de Medellín a través de ciclos de protesta: uno sucedido entre 1996 y 2003 "que marca el inicio de las acciones colectivas de la población desplazada y muestra los motivos de su debilitamiento" (Granada y González, 2009, p. 109), y otro, entre 2004 y 2009, en el que hay nuevos "repertorios de acción" susceptibles de ser identificados "en el discurso de los líderes y organizaciones afectadas por el desplazamiento forzado" (Granada y González, 2009, p. 109). El trabajo también identifica "vacíos en los ejercicios de investigación que indaguen por las personas desplazadas en su rol de sujetos colectivos, capaces de incidir en los escenarios de política pública" (Granada y González, 2009, p. 109).

En cuanto a formas de resistencia, son varias las investigaciones en la ciudad que se han preocupado por comprender los procesos de la acción colectiva y las resistencias en Medellín asociadas a las violencias urbanas. Dos trabajos significativos son los de Jaime Rafael Nieto (2009) y Mary Luz Alzate (2012). Los dos recogen formas de respuestas de la sociedad frente a las violencias, donde han sido fundamentales las "formas de resistencia civil no armada a la luz de experiencias desarrolladas en varios lugares de la ciudad" (Jaramillo, s.f., p. 31), pero también las modalidades de acción política a las que se refiere Alzate en el caso de la comuna 13. De acuerdo con ella, estas acciones

\footnotetext{
[...] crean una política cultural local vinculada a la defensa de la vida, que enfrenta a los violentos y al conflicto armado y democratiza los lugares públicos (las calles, los parques, los callejones, las laderas), recuperándolos para el uso común y libre circulación de todos los pobladores. Se ha aprendido a reconocer que a través de las manifestaciones artísticas y culturales se pueden romper las barreras y los límites bélicos, proponiendo así un nuevo escenario de la política local que reconoce la capacidad del ciudadano de auto-representarse y constituir las condiciones para un bienestar común (Alzate, 2012, pp. 118-119).
}

Esto es fundamental porque se relaciona con las formas que tienen las comunidades, particularmente los jóvenes, de construir nuevas maneras de resistir y 
novedosos procesos del ejercicio ciudadano, tal como señala también la investigadora Deicy Hurtado (2010) para pensarse las formas de expresión política de los jóvenes en Medellín y repensar hipótesis explicativas que señalan que los jóvenes contemporáneos no tienen formas de adscripción política. Este tipo de producciones han sido importantes para visibilizar los procesos de apropiación territorial y de respuestas de la sociedad civil. Sin embargo, una advertencia que hace Mary Luz Alzate es que las acciones colectivas no necesariamente solucionan los problemas sociales articulados al orden violento, sino que "se van configurando y recreando conforme van surgiendo conflictos y alteraciones de los significados compartidos colectivamente" (2012, p. 124).

En cuanto a las resistencias, también es importante anotar que Martin (2014) recoge en su libro sobre Medellín algunas acciones de resistencia generadas desde distintos lugares, tales como la institucionalidad, las ONG y las comunidades locales. Martin resalta cómo, conforme se rearticulan o recomponen distintos procesos violentos, ha habido en la ciudad formas específicas de acción colectiva y de movilización social que proporcionan matices a las violencias urbanas, específicamente a aquellas asociadas al conflicto urbano.

Múltiples iniciativas artísticas, culturales y académicas empezaron a expresarse alrededor de lo acontecido en la ciudad. De acuerdo con Martin, las décadas de 1980 y 1990 se configuran de manera especial por el surgimiento de escenarios como el Centro Colombo Americano, el Festival Internacional de Poesía, diversos espacios musicales, producciones literarias y académicas, entre otras. De hecho, lo narrado por Martin es una continuidad de todos los momentos de la ciudad. En la actualidad, por ejemplo, son múltiples las estrategias culturales de Medellín para narrar desde diferentes orillas y con distintos lenguajes lo que ha pasado en la ciudad. Acá tienen fuerza el teatro, el hip hop, el grafiti, la producción audiovisual, que resignifican no solo la producción de conocimiento y sus estrategias de divulgación, sino también el lugar que ocupan los jóvenes como actores transformadores y posibilitadores del cambio social.

Las investigaciones entre 2010 y 2015 también recogen algunos trabajos sobre desmovilizaciones de los actores armados, tales como los realizados por Vladimir Caraballo (2013), Jorge Giraldo y Juan Pablo Mesa (2013), y Germán Valencia y Leslie Paz (2015). Estos trabajos abordan de manera específica los procesos de desmovilización de las milicias populares de Medellín de los años de 1990, para mostrar cómo estos tuvieron una serie de problemas estructurales y políticos que se reflejan en la reactivación de procesos violentos en la ciudad posterior a estas desmovilizaciones. Los tres textos se concentran en el proceso de desmovilización llevado a cabo en 1994 con las Milicias Populares de Medellín, nombre con el que se agruparon las "organizaciones armadas independientes que hacían presencia en la ciudad desde finales de la década de 1980 - Milicias Populares del Pueblo y para el Pueblo (MP/PP), Milicias Populares Independientes del Valle de Aburrá (MP/IVA) y Milicias Metropolitanas de Medellín (MMM)" (Valencia y Paz, 2015, p. 265).

Caraballo (2013) propone que los procesos de negociación con grupos armados y, particularmente el proceso vivido con las Milicias Populares de Medellín, "deben ser entendidos como mecanismos de relación a través de los cuales los actores de un conflicto ajustan relaciones de poder previamente existentes, y 
no como caminos lineales y altruistas hacia la paz" (p. 244). Esto descentraliza la categoría dicotómica entre guerra y paz y propone otros marcos analíticos para comprender la acción del Estado y de los actores sociales en la negociación el orden social. Para Caraballo (2013) hay que cuestionar el papel del Estado tanto en las confrontaciones con actores armados, así como en los procesos de negociación con estos, ya que no puede concentrarse en otorgar la legitimidad total a los armados, pero tampoco puede militarizar los territorios para retomar su control, tal como sucedió con la Operación Orión en la Comuna 13 (p. 266). La lectura de Caraballo (2013) sobre lo sucedido con las Milicias radica en comprender esto como "una paz híbrida en constante construcción, en la que distintos actores y redes compiten y cooperan a partir de diversos temas y agendas" (p. 268).

Finalmente, otro tema que aparece con fuerza en las publicaciones entre 2010 y 2015 son las lecturas políticas sobre el narcotráfico, y la revitalización de análisis sobre las economías criminales y el crimen organizado en la ciudad. Los trabajos de Gustavo Duncan (2013), Jorge Giraldo (2013), el Centro de Análisis Político EAFIT (2014), el de Giraldo, Rendón y Duncan (2014), así como el texto comparado de Jaramillo y Perea (2014), son muestras de ello. Estos trabajos resignifican las violencias producidas por estos fenómenos a la luz de la violencia política, mostrando continuidades y formas paralelas de actuación que van más allá de manejos económicos del tráfico de drogas, o del control territorial y de rentas. Dichas lecturas posibilitan una mirada histórica más amplia sobre los procesos urbanos e invitan a concebir que la intersección de diferentes tipos de violencias en la ciudad debe leerse bajo un lente político que pueda develar la incidencia de las relaciones de poder en estos fenómenos.

\section{Algunas sugerencias a futuro}

Es evidente que la producción académica sobre la violencia urbana en Medellín se constituye en un enorme potencial para comprender lo que ha sucedido, en la medida en que las hipótesis de los textos, articuladas a las distintas formas de leer las violencias y conflictividades urbanas, resultan fundamentales para conocer matices, posiciones políticas y lecturas analíticas frente a muchos casos coyunturales y procesos de larga duración vividos en el contexto urbano. De hecho, el interés creciente y continuado por la comprensión de las violencias que tienen lugar en Medellín ha invitado a que sectores académicos y sociales repiensen las maneras de producir conocimiento, por lo que la inclusión de métodos variados y la posibilidad de articular diferentes lenguajes narrativos para explicar lo que ha sucedido ha permitido que ciertos grupos y sectores se consoliden como expertos en el tema. Ello, entre otras cosas, ha provocado que los investigadores desarrollen otras maneras de acercarse a sus problemas, revitalizando la producción académica e investigativa.

No obstante, y reconociendo los grandes avances hechos en la ciudad en materia de hipótesis explicativas frente a las violencias, la revisión también evidencia vacíos alrededor de la producción de conocimiento que pueden ser puntos de partida para seguir discutiendo y debatiendo alrededor de las violencias urbanas en Medellín. Al respecto, creemos que algunas de las líneas que se pueden seguir son las siguientes: 
Es necesario abordar con mayor detenimiento aquellos fenómenos violentos que han victimizado grupos poblacionales particulares. Nos referimos de manera específica a mujeres, población victimizada por distintas modalidades -específicamente violencia sexual, masacres y desaparición forzada-, población LGBTI y distintos grupos etarios -niños, niñas, adolescentes, jóvenes y adultos mayores-. Así mismo, las investigaciones asociadas a territorios particulares son un vacío en las producciones. De acuerdo con los estudios identificados, hay una producción importante de trabajos sobre las comunas 1, 3, 8 y 13, pero otros lugares de la ciudad no han sido objeto de análisis. La ausencia más notoria es la de los corregimientos que se han visto afectados directamente por los fenómenos de violencia urbana de la ciudad, pero ellos no han sido objeto de análisis de ninguno de los trabajos académicos identificados.

Así mismo, un elemento importante en cuanto a lo territorial serán trabajos comparados entre comunas en los cuales se identifiquen intensidades de la violencia, patrones y modalidades de victimización, y respuestas de las comunidades de manera diferenciada. La revisión hecha invita a potenciar ciertas formas de trabajo en las investigaciones. Es importante entonces que se sigan fortaleciendo trabajos comparativos -tanto de sectores específicos de la ciudad como con otras ciudades- que posibiliten contrastaciones analíticas y propuestas en conjunto.

Otros sectores sobre los que no se encontraron investigaciones específicas son la fuerza pública y las élites económicas y políticas de la ciudad. En cuanto a la fuerza pública, no fue posible identificar trabajos que mostraran su incidencia o responsabilidad en los hechos acontecidos en Medellín, salvo el Informe de Memoria Histórica para la Comuna 13, los informes de derechos humanos de la Personería de Medellín y los de algunas ONG. Con respecto a las élites, tampoco fue posible identificar ningún trabajo que analizara su incidencia y responsabilidad en los fenómenos violentos, pero tampoco hay investigaciones que evidencien las afectaciones que han sufrido o los aportes realizados. Será interesante que se aborde de manera específica este sector de la población para rastrear las maneras particulares en que las élites se han vinculado con las violencias urbanas, particularmente aquellas asociadas al conflicto armado, y cuáles han sido los sentidos políticos del accionar en estas, máxime si tenemos en cuenta los procesos de negociaciones de paz, en los que se necesitarán conocer las perspectivas de distintos grupos y sectores poblacionales para la construcción de adecuados escenarios de posacuerdo.

Por otro lado, no hay investigaciones concretas que referencien las afectaciones que han sufrido académicos de universidades en la ciudad, particularmente de la Universidad de Antioquia y la Universidad Nacional. Esto es importante porque estas instituciones cargan con un acervo de historias y de hechos violentos que han sucedido dentro de ellas, que bien valdría la pena rastrearlos para reconocer una parte importante de lo acontecido en la ciudad.

Otro campo de análisis importante tiene que ver con el papel de los medios de comunicación acerca de las representaciones que se hacen sobre las violencias urbanas en Medellín. Al respecto, sería interesante tener investigaciones que aborden las representaciones sociales de la violencia en medios locales y nacionales de comunicación, y su articulación con imaginarios culturales sobre actores, causas de la violencia y sus principales efectos, así como trabajos que 
analicen el papel de periodistas en lecturas que se tienen sobre las violencias urbanas, y las implicaciones que tiene trabajar en este tipo de situaciones.

Hay que potenciar también las lecturas sobre las resistencias de las comunidades locales frente a las violencias urbanas y los enfoques de trabajo sobre lo cotidiano, continuando con la agenda de investigación ya iniciada por varios académicos. Estos trabajos, tal como se mostró en páginas anteriores, han aportado relecturas sobre las violencias urbanas y han logrado trazar líneas de análisis entre lo que ocurre a nivel micro y lo que pasa a nivel macro, esto es, han posibilitado análisis escalares donde el centro no necesariamente tiene que estar en lo local o en lo nacional, sino que se evidencia la conexión entre las distintas esferas territoriales. Así mismo, este tipo de lecturas muestran que las situaciones que generan la violencia son móviles y, si se quiere, transitorias.

Por otro lado, hay que seguir potenciando la lectura política de ciertos fenómenos como el narcotráfico y el crimen organizado, así como del accionar paramilitar en la ciudad, lo cual ayudará a establecer conexiones entre los distintos tipos de violencia presentes en Medellín que, se intuye, se congregan de manera particular en la violencia política. Esto, además, podrá ayudar a esclarecer la trashumancia, continuidad y aprendizajes de los actores armados que han hecho presencia en Medellín desde finales de la década de 1970.

De cara a la coyuntura que vive el país con las negociaciones de paz y la posible implementación de los acuerdos a mediano y largo plazo, es necesario seguir pensando cómo definimos nuestros objetos de investigación, las preguntas que nos hacemos y los métodos que empleamos; es decir, preguntarnos qué significa investigar en medio de la violencia y qué limitaciones se nos imponen en el ejercicio académico e investigativo. Así mismo, es importante revisar qué papel juega el sector académico en el marco del actual posacuerdo: ¿será necesario que todas las investigaciones miren hacia el futuro? $\mathrm{O}$, por el contrario, ¿es importante revisar lo dicho y seguir explorando lo que ha pasado en materia de violencias urbanas para lograr efectivas garantías de no repetición? Es en este último punto donde, consideramos, debe enfocarse una parte de nuestro trabajo como investigadores, pues la transición que se vislumbra seguramente dará otras perspectivas para repensar las hipótesis con las que hemos leído tradicionalmente las violencias urbanas.

También se hace necesario revelar con mayor fuerza los imponderables metodológicos a los que nos enfrentamos de manera cotidiana en los trabajos y en los que los silencios cumplen un papel fundamental (Quiceno, 2008). Esto es, no es solo la víctima quien calla lo acontecido, sino que los investigadores, por lo difícil de su ejercicio y también por las implicaciones éticas y políticas de lo dicho, han tenido que callar en estos años de historia. Seguramente, en décadas siguientes se aportarán elementos ahora soterrados que cambien el rumbo del trabajo investigativo; pero, por ahora, es necesario hacer evidente que trabajamos en medio de la violencia y que nuestras palabras, como ya anotaba María Teresa Uribe de Hincapié (2002), tienen responsabilidad e injerencia en estas.

La invitación entonces es a seguir haciendo visible ese lugar de enunciación del investigador, y a reconocer crítica y reflexivamente el trabajo realizado, más aún cuando se tiene la oportunidad de repensar las hipótesis que se han construido sobre lo acontecido en Medellín. Es importante que se aborde el impacto 
que han tenido las palabras de la investigación en la ciudad, y que se revisen los ejercicios de memorias y de reparación realizados hasta ahora, con el fin de mantener vivo el debate y abierta la posibilidad de discusión y reflexión. Esto para no olvidar el papel que las ciudades tendrán en el futuro posacuerdo y lo necesario que sigue siendo pensar aquello que hemos dicho y la forma como hemos analizado los tiempos de guerra, esto con el fin de construir escenarios adecuados para la paz.

\section{Referencias}

Alonso, M., Giraldo, J. y Sierra, D. (2006). Medellín: el complejo camino de la competencia armada. En C. de Gamboa (Ed.), Justicia transicional: teoría y praxis (pp. 435-465). Bogotá, Colombia: Universidad del Rosario.

Alonso, M., Pérez, W. y Vélez, J. C. (2012). Ensayos sobre conflicto, violencia y seguridad ciudadana en Medellín, 1997-2007. Medellín, Colombia: Universidad de Antioquia.

Alzate, M. L. (2012). Acciones colectivas frente a la violencia. Disquisiciones a partir de un estudio de caso: Comuna 13 de Medellín (Colombia). FORUM, 1(3), 111-130.

Arenas, S. (2012). Memorias que perviven en el silencio. Universitas Humanística, (74), $173-193$.

Berrío, A., Grisales, M. y Osorio, R. (2011). Violencia y subjetividad: narrativas de la vida cotidiana. Medellín, Colombia: Universidad de Antioquia.

Blair, E., Quiceno, N., De los Ríos, I., Muñoz, A., Grisales, M. y Bustamante, G. (2008). De memorias y de guerras. La Sierra, Villa Liliam y el 8 de marzo en Medellín. Informe de investigación. Medellín, Colombia: Alcaldía de Medellín; INER.

Blair, E., Grisales, M. y Muñoz, A. M. (2009). Conflictividades urbanas vs. "Guerra urbana" Otra "clave" para leer el conflicto en Medellín. Universitas Humanística, 67(67), 29-54.

Caraballo, V. (2013). Órdenes locales, acuerdos de paz y presencia diferenciada del Estado. Negociación con las Milicias Populares de Medellín. Colombia Internacional, (77), 241-270.

Centro de Análisis Político EAFIT. (2014). Acompañamiento al diseño de política pública de seguridad y convivencia para el municipio de Medellín. Informe de investigación. Medellín, Colombia: EAFIT.

Centro Nacional de Memoria Histórica. (2015). Limpieza social. Una violencia mal nombrada. Bogotá, Colombia: CNMH - IEPRI.

Centro Nacional de Reparación y Reconciliación. (2011). Desplazamiento forzado en la comuna 13. La huella invisible de la guerra. Medellín, Colombia: CNRR.

Díaz, V., Molina, A. y Marín, M. (2015). Las pérdidas y los duelos en personas afectadas por el desplazamiento forzado. Pensamiento psicológico, 13(1), 65-80.

Duncan, G. (2013). Una lectura política de Pablo Escobar. Co-herencia, 10(19), 235-262.

Franco, V. (2000). Sobre los estudios frente al conflicto urbano en Medellín; algunas consideraciones generales. En J. W. Balbín (Comp.), Movimiento Social por la Paz y Hermanamientos. Pueblos Hermanos... Lazos Visibles (pp. 83-118). Medellín, Colombia: IPC. 
García, C. (2011). Los estudios sobre órdenes locales. Enfoques, debates y desafíos. Análisis Político, 24(73), 55-78.

Gil, M. (2009). Paramilitarismo y conflicto urbano: relaciones entre el conflicto armado nacional y las violencias preexistentes en la ciudad de Medellín: 19972005 (tesis de maestría). Universidad de Antioquia, Medellín, Colombia.

Giraldo, J. y Mesa, J. P. (2013). Reintegración sin desmovilización: el caso de las Milicias Populares de Medellín. Colombia Internacional, (77), 217-239.

Giraldo, J. (Ed.). (2013). Economía criminal y poder político. Medellín, Colombia: EAFIT; Colciencias.

Giraldo, J., Rendón, A. y Duncan, G. (2014). Nuevas modalidades de captación de rentas ilegales en Medellín. Medellín, Colombia: EAFIT; ESU; Alcaldía de Medellín.

Granada, J. y González, S. (2009). Acción colectiva de las organizaciones de población desplazada en Medellín. Ciclos, contextos, repertorios y perspectivas. Estudios Políticos, (35), 107-130.

Hurtado, D. (2010). Los jóvenes de Medellín: ¿Ciudadanos apáticos? Nómadas, (32), 99-115.

Jaramillo, A. M. (s.f.). Acerca de los estudios sobre conflicto armado y violencia urbana en Medellín (1985-2009). Recuperado de http://www.region.org.co/ images/publicamos/documentos/Acerca_de_los_estudios_sobre_conflicto_ armado_y_violencia_urbana_en_Medellin.pdf

Jaramillo, A. y Perea, C. M. (2014). Ciudades en la encrucijada: Violencia y poder criminal en Río de Janeiro, Medellín, Bogotá y Ciudad Juárez. Medellín, Colombia: Corporación Región; IEPRI; UNAL; IDRC.

Martin, G. (2014). Medellín, tragedia y resurrección: mafia, ciudad y Estado: 19752012. Medellín, Colombia: La Carreta Editores.

Molina, A., Díaz, V. y Marín, M. (2014). Significación de la experiencia de pérdidas y duelos en personas desplazadas de la ciudad de Medellín. Informe de investigación. Medellín, Colombia: Facultad de Ciencias Sociales y Humanas, Universidad de Antioquia.

Murcia, J. (2010). Acción colectiva de la población en situación de desplazamiento en Medellín y su reivindicación del derecho a la ciudad: análisis del período 2000-2009 (tesis de pregrado). Universidad de Antioquia, Medellín, Colombia.

Nieto, J. y Robledo, L. J. (2006). Conflicto, violencia y actores sociales en Medellín. Zona centroriental comunas 8 y 9. Medellín, Colombia: Universidad Autónoma Latinoamericana; EPM.

Nieto, J. (2009). Resistencia civil no armada en Medellín. La voz y la fuga de las comunidades urbanas. Análisis Político, 22(67), 38-59.

Nieto, P. (2013). Relatos autobiográficos del conflicto armado en Colombia. El caso reciente de la ciudad de Medellín (tesis de doctorado). Universidad Nacional de La Plata, La Plata, Argentina.

Perea, C. M. (2014). La muerte próxima: Vida y dominación en Río de Janeiro y Medellín. Análisis Político, 27(80), 3-38.

Perea, C. M. (2016). Vislumbrar la paz: Violencia, poder y tejido social en ciudades latinoamericanas. Bogotá, Colombia: Penguin Random House; Universidad Nacional. 
Pérez, A., Aristizábal, C., Ríos, D. y Osorno, Y. (2014). Construcción de ciudad: entre los filos de la memoria y la violencia. Caso Manrique, Medellín. Estudios Políticos, (44), 141-161.

Quiceno, N. (2008). Puesta en escena, silencios y momentos del testimonio. El trabajo de campo en contextos de violencia. Estudios Políticos, (33), 181-208.

Restrepo, W., Pérez, W. y Vélez, J. C. (2012a). Aproximación al estudio de la violencia urbana en Colombia y de la criminalidad homicida en Medellín. En M. Alonso, W. Pérez y J. C. Vélez (Eds.), Ensayos sobre conflicto, violencia y seguridad ciudadana en Medellín, 1997-2007 (pp. 15-52). Medellín, Colombia: Universidad de Antioquia.

Restrepo, W., Pérez, W. y Vélez, J. C. (2012b). La criminalidad homicida en Medellín 1986-1996. En M. Alonso, W. Pérez y J. C. Vélez (Eds.), Ensayos sobre conflicto, violencia y seguridad ciudadana en Medellín, 1997-2007 (pp. 87-124). Medellín, Colombia: Universidad de Antioquia.

Riaño, P. (2006). Jóvenes memoria y violencia en Medellín. Una antropología del recuerdo y el olvido. Medellín, Colombia: Universidad de Antioquia.

Secretaría de la Juventud. (2015). Carrera de obstáculos. Crecer y resistir. Relatos sobre seguridad y convivencia juvenil en Medellín. Recuperado de http://issuu. com/medellinjoven/docs/digital/1

Tabares, C. (2011). Reflexiones en torno al devenir sujeto político de las víctimas del conflicto armado. Estudios Políticos, (38), 13-37.

Universidad del Rosario. (2012). 74\% de la población colombiana habita en zonas urbanas. Recuperado de http://www.mineducacion.gov.co/cvn/1665/w3article-300919.html

Uribe-de-Hincapié, M. T. (2002). La investigación social en tiempos de guerra. Utopía Siglo XXI, 2(8), 13-22.

Valencia, G., y Paz, L. (2015). Atipicidades del proceso de paz con las Milicias Populares de Medellín. Estudios Políticos, (46), 263-282.

Vergara, M. (2013). Gobernabilidades en vilo: violencia y regulación en la Medellín urbana. Diversas interpretaciones. Recuperado de http://actacientifica.servicioit.cl/biblioteca/gt/GT4/GT4_VergaraArias.pdf

Villegas, D. (2014). Seguridad y control 1968-1969 (tesis de pregrado). Universidad de Antioquia, Medellín, Colombia.

Yarce, C. (2013). Militarización de la seguridad ciudadana en Medellín. Estudios de Derecho, 70(156), 266-290.

Zapata, C. (2012). Las formas expresivas de la violencia en Colombia. En IPC (Ed.), Conflicto y formas expresivas de la violencia en contextos situados: aproximación a cuatro territorios de Antioquia (pp. 7-84). Medellín, Colombia: IPC. 
